This item was submitted to Loughborough's Research Repository by the author.

Items in Figshare are protected by copyright, with all rights reserved, unless otherwise indicated.

\title{
Optimal selection for sensor fault tolerant control of an EMS system via loop- shaping robust control
}

PLEASE CITE THE PUBLISHED VERSION

http://dx.doi.org/10.1109/MED.2011.5983065

PUBLISHER

(C) IEEE

VERSION

VoR (Version of Record)

LICENCE

CC BY-NC-ND 4.0

\section{REPOSITORY RECORD}

Michail, Konstantinos, Argyrios C. Zolotas, Roger M. Goodall, and G.D. Halikias. 2019. "Optimal Selection for Sensor Fault Tolerant Control of an EMS System via Loop-shaping Robust Control”. figshare. https://hdl.handle.net/2134/9256. 
This item was submitted to Loughborough's Institutional Repository (https://dspace.lboro.ac.uk/) by the author and is made available under the following Creative Commons Licence conditions.

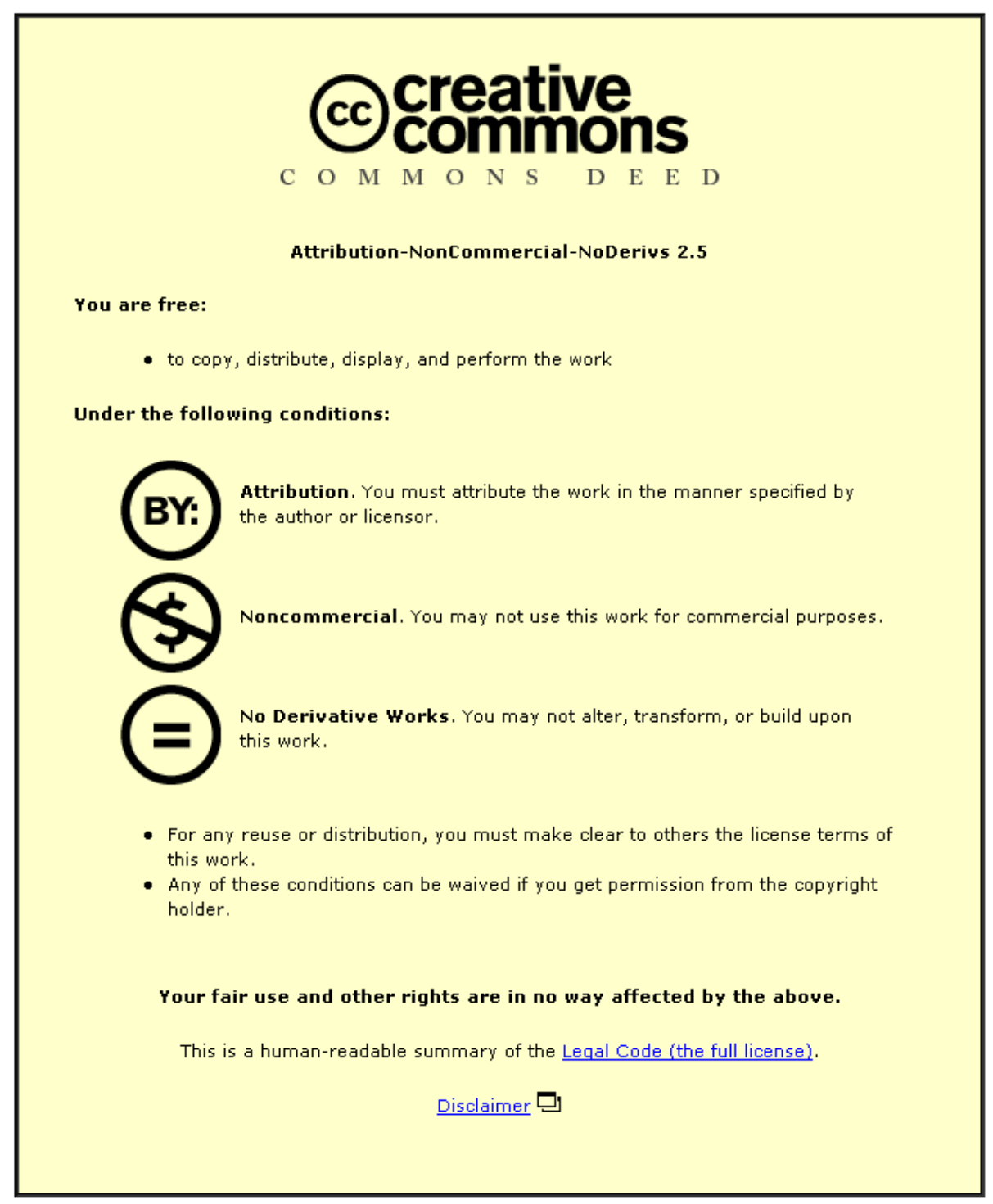

For the full text of this licence, please go to: http://creativecommons.org/licenses/by-nc-nd/2.5/ 


\title{
Optimal Selection for Sensor Fault Tolerant Control of an EMS System via Loop-Shaping Robust Control
}

\author{
Konstantinos Michail, Argyrios C Zolotas, Roger M Goodall and George Halikias
}

\begin{abstract}
A systematic framework is presented for optimum sensor selection for control and fault tolerance subject to complex system requirements. The framework combines the well known robust control via loop-shaping design, the fault tolerance control concept and multiobjective optimisation. The framework is tested via realistic simulations on an ElectroMagnetic Suspension system which is a non-linear, unstable and safety-critical system with a set of non-trivial requirements.
\end{abstract}

\section{INTRODUCTION}

Optimum selection of sensors is not a trivial task especially if a lot of candidate sensors exist. The problem becomes more complicated when a lot of conflicting closedloop objectives are to be met like optimum performance, robustness and sensor fault tolerance with the minimum possible number of sensors. The proposed systematic framework actually proves to simplify the sensor selection process subject to the aforementioned control properties. The proposed framework has been tested under various modern control methods including Linear Quadratic Gaussian (LQG) control [17], [19], Multiobjective $\mathcal{H}_{\infty}$ robust control [15], [18] and the $\mathcal{H}_{\infty}$ Loop-Shaping Design Procedure (LSDP) [16], [20]. The later publication in [20] includes sensor selection for control but without considering optimum sensor selection for fault tolerant control and the non-linearities of the EMS system that concern this paper. Thus, the systematic framework is extended towards optimum sensor selection for fault tolerance control of the non-linear EMS system by using the LSDP. Combining the loop-shaping design as presented in [13], the well known fault tolerant concept [1], [2] and the powerful Genetic Algorithms (GA) [11] that are extensively used in control engineering design optimisation [5] the framework is formed.

The Electro-Magnetic Suspension (EMS) systems are being used on the MAGLEV trains for both supporting the payload of the vehicle as well as ensure proper ride quality at high velocities replacing conventional wheel trains that have a number of disadvantages [12]. As indicated in [8] the EMS

The Authors would like to thank Engineering and Physical Sciences Research Council, UK, for supporting this research work under the project Grand Ref. EP/D063965/1, and in part under the NEW-ACE project ref. EP/E055877/1, and BAE Systems from Systems Engineering Innovation Centre, UK.

K. Michail is with the Energy, Environment and Water Research Center, The Cyprus Institute, Nicosia, Cyprus. kon_michael@ieee.org

A.C.Zolotas and R.M.Goodall are with Control Systems Group, Department of Electronic and Electrical Engineering, Loughborough University, Loughborough, UK \{a.c.zolotas, r.m.goodall\} @lboro.ac.uk

G.Halikias is with Control Engineering Research Centre, School of Engineering and Mathematical Sciences, City University, London, UK g.halikias@city.ac.uk system is a non-linear, inherently unstable with a set of non-trial requirements. Running at high speeds with a large payload the control system of an EMS has to guarantee its performance and stability under sensor faults which makes it (on top of the others) a safety-critical system. Thus the EMS can easily serve as a good example for testing the efficacy of the proposed framework.

This paper is separated into five sections: Section 2 describes the EMS model and closed-loop requirements. In Section 3 the details of the proposed sensor optimisation framework are given and in Section 4 the simulation results are discussed and the efficacy of the proposed framework is assessed. The paper concludes by summarizing the advantages in Section 5 .

\section{SINGLE STAGE EMS AND TRACK MODELS}

\section{A. The EMS Model}

The single-stage EMS system model represents the quarter of a typical MAGLEV vehicle and is given here. The details of the model for such a suspension are extensively discussed in [14] hence not details are given in this section.

The non-linear model of the EMS is described by Newton's equation of motion and the Kirchhoff's law and is given as

$$
\begin{gathered}
\frac{d I}{d t}=\frac{V_{c}-I R_{c}+\frac{N_{c} A_{p} K_{b}}{G^{2}}\left(\frac{d z_{t}}{d t}-\frac{d Z}{d t}\right)}{\frac{N_{c} A_{p} K_{b}}{G}+L_{c}} \\
\frac{d^{2} Z}{d^{2} t}=g-\frac{K_{f}}{M_{s}} \frac{I^{2}}{G^{2}} \\
B=K_{b} \frac{I}{G}, F=K_{f} B^{2} \\
\frac{d G}{d t}=\frac{d z_{t}}{d t}-\frac{d Z}{d t}
\end{gathered}
$$

where $V_{c}$ is the coil's voltage, $F$ the vertical force, $I$ the coil's current, $G$ the airgap, $Z$ the electromagnet's and $z_{t}$ the track's position.

Constants $K_{b}, K_{f}$ and $g$ are the flux, force and gravity constants given as $0.0015,0.0221$ and $9.81 \mathrm{~m} / \mathrm{s}^{2}$ respectively. Other symbols in Eqs. (1)- (4) are the EMS parameters listed on Table I.

The linearisation of the non-linear model is based on small perturbations around the operating point. i.e. the air gap is taken as $G=G_{o}+\left(z_{t}-z\right)$ with lower case letter defining the small variation around the operating point and subscript 'o' referring to the operating point. Similar approach is done for $B, F, I, V_{c}$ and $Z$. 
Following the linearization procedure in [8] the state space description of the EMS can be expressed in state space form as in (5) where the selected states are $x=\left[\begin{array}{lll}i & \dot{z} & \left(z_{t}-z\right)\end{array}\right]^{T}$ and the output equation corresponds to the following five measurements: $i$, the coil's current, $b$ the flux density, $\left(z_{t}-z\right)$ is the air gap, $\dot{z}$ the vertical velocity and $\ddot{z}$ the vertical acceleration. The matrices $A, B_{u_{c}}, B_{\dot{z}_{t}}$ and $C$ are given by (6)-(8).

$$
\begin{aligned}
& \dot{x}=A x+B_{u_{c}} u_{c}+B_{\dot{z}_{t}} \dot{z}_{t} \\
& y=C x \\
& A=\left[\begin{array}{ccc}
-\frac{R_{c}}{L_{c}+\frac{K_{b} N_{c} A_{p}}{G_{o}}} & -\frac{K_{b} N_{c} A_{p} I_{o}}{G_{o}^{2}\left(L_{c}+\frac{K_{b} N_{c} A_{p}}{G_{o}}\right)} & 0 \\
-2 K_{f} \frac{I_{o}}{M_{s} G_{o}^{2}} & 0 & 2 K_{f} \frac{I_{o}^{2}}{M_{s} G_{o}^{3}} \\
0 & -1 & 0
\end{array}\right] \\
& B_{u_{c}}=\left[\begin{array}{c}
\frac{1}{L_{c}+\frac{K_{b} N_{c} A_{p}}{G_{o}}} \\
0 \\
0
\end{array}\right], B_{\dot{z}_{t}}=\left[\begin{array}{c}
\frac{K_{b} N_{c} A_{p} I_{o}}{G_{o}^{2}\left(L_{c}+\frac{K_{b} N_{c} A_{p}}{G_{o}}\right)} \\
0 \\
1
\end{array}\right] \\
& C=\left[\begin{array}{ccc}
1 & 0 & 0 \\
\frac{K_{b}}{G_{o}} & 0 & -\frac{K_{b} I_{o}}{G_{o}^{2}} \\
0 & 0 & 1 \\
0 & 1 & 0 \\
-2 K_{f} \frac{I_{o}}{M_{s} G_{o}^{2}} & 0 & 2 K_{f} \frac{I_{o}^{2}}{M_{s} G_{o}^{3}}
\end{array}\right]
\end{aligned}
$$

Output matric, $\mathrm{C}$ gives the five measurements (i.e. $i, b,\left(z_{t}-\right.$ $z), \dot{z}$ and $\ddot{z}$ ) and the sensor combinations can be obtained by using the corresponding rows of $C$ in (8). The total number of sensor sets, $N_{s}$ is given as $N_{s}=2^{n_{s}}-1$. Where $n_{s}$ is the total number of sensors. Given that the EMS system has 5 outputs there are 31 candidate sensor sets. However, since the LSDP controller design technique is used here the air gap measurement is a standard measurement and the candidate sensor sets reduces to 16 .

Details for EMS design of MAGLEV vehicles are given in [9] but is out of the scope of this paper. However the operating point along with the electromagnet's parameters are tabulated on Table I.

TABLE I

\begin{tabular}{llll}
\multicolumn{4}{c}{ PARAMETERS OF THE EMS SYSTEM. } \\
\hline \hline \multicolumn{1}{c}{ Parameter } & \multicolumn{1}{c}{ Value } & \multicolumn{1}{c}{ Parameter } & \multicolumn{1}{c}{ Value } \\
\hline Op. air gap, $G_{O}$ & $0.015 \mathrm{~m}$ & Carriage Mass, $M_{s}$ & $1000 \mathrm{~kg}$ \\
Op. flux density, $B_{O}$ & $1 \mathrm{~T}$ & Coil's Resistance, $R_{c}$ & $10 \Omega$ \\
Op. current, $I_{O}$ & $10 \mathrm{~A}$ & Coil's Inductance, $L_{c}$ & $0.1 \mathrm{H}$ \\
Op. voltage, $V_{o}$ & $100 \mathrm{~V}$ & Number of turns, $N_{c}$ & 2000 \\
Op. force, $F_{o}$ & $9810 \mathrm{~N}$ & Pole face area, $A_{p}$ & $0.01 \mathrm{~m}^{2}$ \\
\hline \hline
\end{tabular}

Note: Op. stands for Operating.

\section{B. The EMS Control Requirements and Disturbance Inputs}

1) Stochastic Inputs: The stochastic inputs are random variations of the rail position as the vehicle moves along the track. This is caused by the steel rail installation discrepancies, due to rail-laying inaccuracies as well as unevenness off the rail. Considering the vertical direction, the velocity variations can be approximated by a double-sided power spectrum density (PSD) expressed as $S_{\dot{z}_{t}}=\pi A_{r} V_{v}$. The $V_{v}$ is the vehicle speed (taken as $15 \mathrm{~m} / \mathrm{s}$ in this case) and $A_{r}$ represents the rail's roughness and it is assigned a value of $1 \times 10^{-7}$ that is used for high quality rail. Then the corresponding autocorrelation function is given as $R(\tau)=$ $2 \pi^{2} A_{r} V_{v} \delta(\tau)$. Although the LSDP is linear controller, the simulations are actually based on the implementation to the nonlinear model. Hence, the RMS values of the required quantities (acceleration, current etc) are calculated using time history data.

2) Deterministic Input: The main deterministic input to the suspension in the vertical direction is due to the transition onto a gradient. In this work, the deterministic input is a rail gradient of $5 \%$ at a vehicle speed of $15 \mathrm{~m} / \mathrm{s}$, an acceleration of $0.5 \mathrm{~m} / \mathrm{s}^{2}$ and a jerk of $1 \mathrm{~m} / \mathrm{s}^{3}$ [14].

3) EMS Control Properties: The design requirements for an EMS system depend on the type and speed of the train. and they are well described by Goodall $(1994,2004)$ [6], [7]. His work is focused upon the low speed Birmingham airport MAGLEV vehicle EMS requirements which operated successfully in the UK for a period of 12 years in the 1980s and 1990s. The EMS has to be able to support the payload i.e. the passengers while reject the stochastic inputs (i.e. randomness due to track irregularities) and follow the deterministic ones (i.e. track gradients). Fundamentally, there is a trade-off between the deterministic and stochastic responses and there are some limitations that they are allowed to operate tabulated in Table II. The deterministic features are limited to the maximum standard values and the stochastic ones are set as objectives to be minimized i.e. the vertical acceleration $\ddot{z}_{r m s}$ (improve ride quality) and the RMS current variations $i_{r m s}$ from the stochastic response. The robust stability margin $\epsilon$ calculated from the LSDP is maximized for maximum robustness to uncertainties (note that $\gamma=1 / \epsilon$ ).

Since noise affects the sensors, an amount of this noise will appear on the control effort $u_{n_{r m s}}$ [19]. In that case the noise can be amplified from the controller if not considered hence it is minimized as well. Summarizing, the objective functions $\phi_{i}$ to be minimized are formally written as:

$$
\phi_{1}=i_{r m s}, \phi_{2}=\gamma, \phi_{3}=\ddot{z}_{r m s}, \phi_{4}=u_{n_{r m s}}
$$

\section{THE SENSOR OPTIMISATION FRAMEWORK}

The proposed framework can be summarised in the flow chart of Fig. 1. The particular points include the use of $\mathcal{H}_{\infty}$ loop-shaping design and the GA method for optimal tuning of the closed-loop response subject to strict requirements (objectives and constraints) for each feasible sensor set of 
TABLE II

CONSTRAINTS ON THE EMS SYSTEM PERFORMANCE.

\begin{tabular}{l|l}
\hline \hline \multicolumn{1}{c|}{ EMS limitations } & \multicolumn{1}{c}{ Value } \\
\hline RMS acceleration, $\ddot{z}_{r m s}$ & $\leq 1 \mathrm{~ms}^{-2}$ \\
RMS air gap variation, $\left(z_{t}-z\right)_{r m s}$ & $\leq 5 \mathrm{~mm}$ \\
RMS control effort, $u_{c_{r m s}}$ & $\leq 300 V\left(3 I_{0} R_{c}\right)$ \\
Maximum air gap deviation, $\left(z_{t}-z\right)_{p}$ & $\leq 7.5 \mathrm{~mm}$ \\
Control effort, $u_{c_{p}}$ & $\leq 300 V\left(3 I_{0} R_{c}\right)$ \\
Settling time, $t_{s}$ & $\leq 3 s$ \\
Air gap Steady state error, $\left(z_{t}-z\right)_{e_{s s}}$ & $=0$ \\
Robust Stability Margin, $\epsilon$ & $\geq 0.15$ \\
\hline \hline
\end{tabular}

the EMS. The type of GA used is the so called NonDominated Sorting Genetic Algorithm II (NSGAII) developed by Deb(2002)[4].

Prior to running the algorithm (initialization phase), some parameters are assigned including thee GA parameters, define the objective functions (9), the constraints of the closed-loop response as listed in Table II,the controller selection criteria $\left(f_{c_{i}}\right)$ and the user's controller selection criterion $\left(f_{k}\right) . f_{c_{i}}$ and $f_{k}$ make sure that the selected controller results in a desired closed-loop performance.

Starting the optimisation procedure, the first sensor set is selected and the GA seeks for the Pareto-optimality of the objective functions in (9) (i.e. the trade-off between the functions in (9)) subject to the constraints listed in Table II. In the sequence, the algorithm seeks to find the optimized

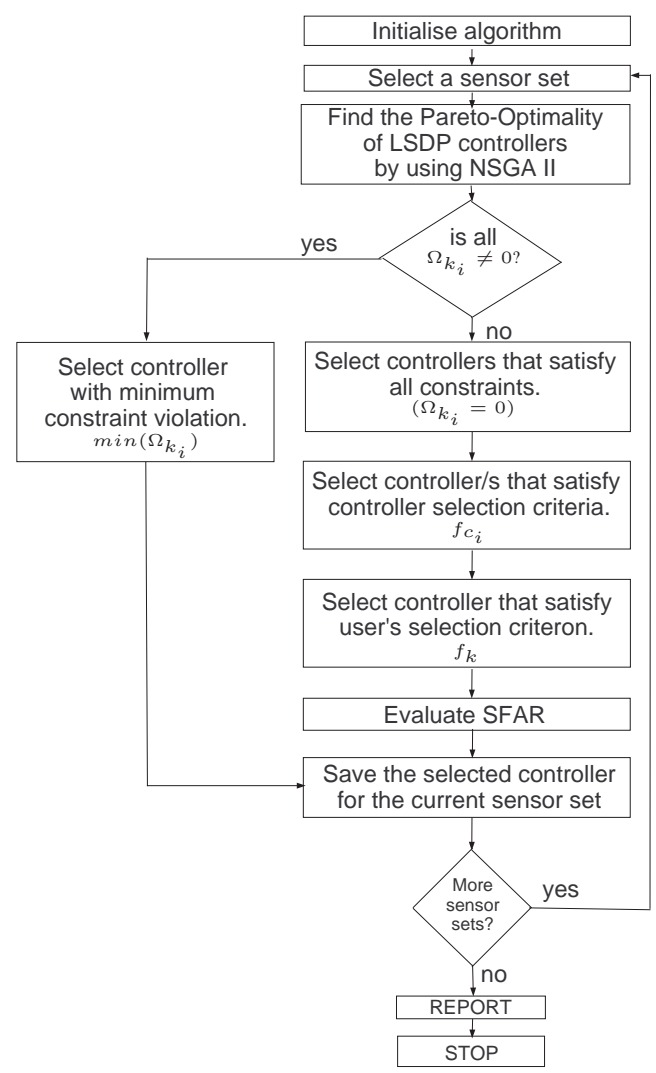

Fig. 1. Flow chart of the systematic framework.
LSDP designed controller by using the overall constraint violation function, $\Omega$ (see (13) in Section III-C). At this point there are two paths to follow:

(i) If there is no sufficient controller (this can be easily verified by checking $\Omega$ for each individual response) then the controller which gives the minimum $\Omega$ is selected and saved. After that the selected optimally tuned controller is saved and the algorithm moves to the next feasible sensor set.

(ii) Those controllers satisfying $\Omega$ are selected and those controllers that satisfy the controller selection criteria $f_{c_{i}}$ are taken for further consideration. Finally, the user's controller selection criteria, $f_{k}$ is used to select the controller which results in the desired closed-loop response. The optimally tuned controller is saved and the algorithm moves to the next stage where the evaluation of the selection criterion for optimum sensor fault tolerance is done. At this stage the Sensor Fault Accomodation Ratio (SFAR) is evaluated which indicates at which extend the faults can be recovered in sense of the number of fault conditions that could be accommodated by using the remaining healthy sensors in a given optimum sensor set $\mathcal{Y}_{\text {opt }}$.

Assuming that $\mathcal{Y}_{o p t}$ is the optimum sensor set to be used then all possible sensor fault combinations are subsets of that sensor set i.e $\mathcal{Y}_{o p t_{f}} \subset \mathcal{Y}_{\text {opt }}$ (assuming that is not possible to loose all sensors). Then, $\mathcal{Y}_{o p t_{h}}$ is the healthy sensor sets that can successfully be used for maintaining the performance under any sensor fault combination (this can be easily identified from $\Omega$ for each of the sensor sets). Thus, the SFAR is formally defined as

$$
S F A R=\frac{N_{\mathcal{Y}_{\text {opt }_{h}}}}{N_{\mathcal{Y}_{\text {opt }_{f}}}} 100(\%)
$$

where the $N_{\mathcal{Y}_{o p t_{h}}}$ and $N_{\mathcal{Y}_{o p t_{f}}}$ are the number of the sensor sets in $\mathcal{Y}_{o p t_{h}}$ and $\mathcal{Y}_{o p t_{f}}$ respectively. This metric is used for optimum sensor set selection that offers the highest fault tolerance against sensor failures.

Finally, the algorithm moves to the next feasible sensor set until all sensor sets are checked as described above.

Following the EMS modelling consideration and requirements the rest of the tools forming the core of the proposed framework are described.

\section{A. $\mathcal{H}_{\infty}$ Loop-shaping Robust Control Design}

The design of the controller is based on the normalised coprime-factor plant description, proposed by [13], which incorporates the simple performance/robustness tradeoff obtained in loop shaping, with the normalised Left Coprime (LCF) robust stabilization method as a means of guaranteeing closed-loop stability.

The design method proceeds by shaping the open-loop characteristics of the plant by means of the weighting functions $W 1$ and $W 2$ (see Fig. 2(a)). The plant is temporarily redefined as $\hat{G}(s)=W 2 G W 1$ and the $\mathcal{H}_{\infty}$ optimal controller $\hat{K}(s)$ is calculated. In the final stage, the weighting functions are merged with the controller by defining the overall controller $K(s)=W 1 \hat{K} W 2$ as shown in Fig. 2(b). 
The size of model uncertainty is quantified by the stability

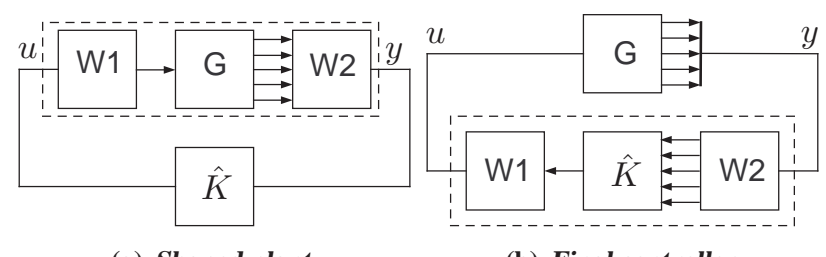

(a) Shaped plant.

(b) Final controller.

Fig. 2. $\mathcal{H}_{\infty}$ loop-shaping design.

radius $\epsilon$ (refer to [13] and [22] for more details), i.e. the stability margin. For values of $\epsilon>0.25,25 \%$ coprime factor uncertainty is allowable. However, in this paper a relaxed constraint is used to have an $\epsilon \geq 0.15$ instead.

In typical design the filter functions and thus the controller are to be kept as simple as possible. Thus, the $W 1$ precompensator, is chosen as a single scalar weighting function set to unity. For the $W 2$ post-compensators there can be five weighting functions that are used depending on the selected sensor set. The airgap $\left(z_{t}-z\right)$ measurement is a compulsory measurement required for proper maglev control of the magnet distance from the rail and thus a low pass filter $\left(W_{\left(z_{t}-z\right)}\right)$ is chosen with integral action allowing zero steady state airgap error (for the nominal performance). The weighting functions are given as

$$
W 1=1 ; \quad W 2=\operatorname{diag}\left(W_{i}, W_{b}, W_{\left(z_{t}-z\right)}, W_{\dot{z}}, W_{\ddot{z}}\right)
$$

with,

$$
W_{\left(z_{t}-z\right)}=\left(\frac{\frac{s}{M_{p}^{1 / n}}+\omega_{b}}{s+\omega_{b} A_{p}^{1 / n_{p}}}\right)^{n_{p}}
$$

The low pass filter results to a minimum phase and stable weighting filter with roll-off rate $n_{p}$. Details for weighting function selection can be found in [22].

\section{B. Sensor Fault Tolerance Scheme}

Fault tolerance is a subject that has been a main point of research studies in the last years [1], [2]. In this paper the aim is to recover the stability and performance in an optimum manner under multiple sensor failures. Under this scope the Active Fault Tolerant Control (AFTC) concept is used [21], [23]. The AFTC concept is composed from a bank of $\mathcal{H}_{\infty}$ designed controllers. When multiple sensor faults happen remedial actions are taken by controller reconfiguration. The recovery of the performance is aimed by using the remaining healthy sensors (sub-set of the selected sensor set) as depicted in Fig. 3. The reconfiguration scheme requires a Fault Detection and Isolation (FDI) mechanism in order to detect and isolate the faulty sensors while it produces the controller reconfiguration signal. Note that the switching delay is assumed to be negligible for simplicity. Typically, a common way to detect a fault is to monitor the residual of two signals. The residuals for each output is typically produced by means of dedicated observers [10]. A bank of dedicated observers (i.e. $K_{o_{1}}, K_{o_{2}} \ldots K_{o_{n}}$ ) is used to monitor

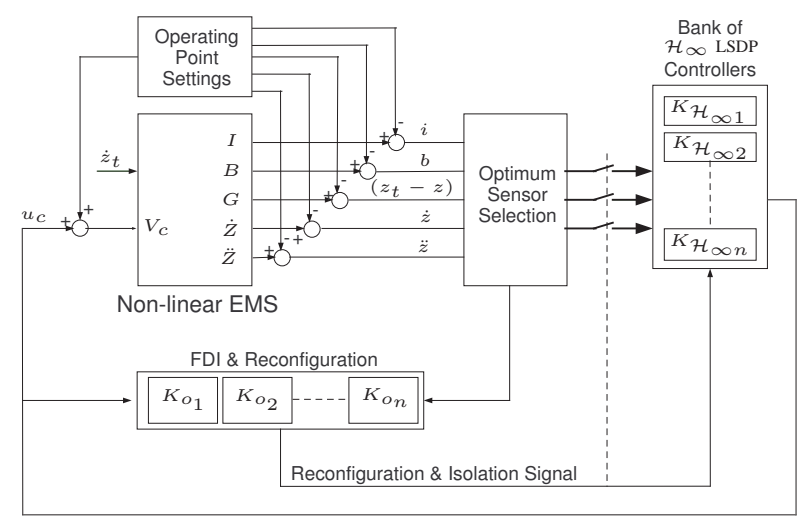

Fig. 3. AFTC diagram for multiple sensor failures using a bank of $\mathcal{H}_{\infty}$ LSDP controllers.

the condition of each sensor as depicted in Fig. 3. Isolating the faulty sensors is done by taking the sensor out of the loop in such a way that the faulty signal is not fed to the new controller (i.e. switching). Sensor faults modelling can be done in three ways [10]: (i) abrupt fault (stepwise) (i)incipient fault (drift-like) and (iii)intermittent fault. In this paper the first sensor fault model is considered.

\section{Multi-objective Constrained Optimisation}

Heuristic approaches are very powerful optimisation tools that are implemented in many engineering problems and particularly GAs have been extensively implemented in control engineering [5]. Different types of GAs have been developed the last years and they are well summarized in [11]. In this work, the recently developed GA based on non-dominated sorting of the population, Non-Dominated Sorting Genetic Algorithm II (NSGA-II) is used that proofs to be a powerful optimization tool within the proposed framework [4].

NSGA-II is an evolutionary process that is based on genetic operators like the crossover and mutation. These requires some parameters to be assigned in order to ensure proper population convergence towards Pareto-Optimality and they are mainly selected from experience rather than from apriori knowledge of the optimisation problem. The crossover probability is generally selected to be large in order to have a good mix of genetic material. The crossover probability is set to $90 \%$ and the mutation probability is defined as $1 / n_{u}$ where, $n_{u}$ is the number of variables. The population consists of 50 chromosomes and the stopping criterion is the maximum generation number $N_{g e n}$. $N_{\text {gen }}$ has a significant role on the Pareto-Optimality and the computational time i.e. the higher the generation number is the longer the computational time but it is more possible for the evolved population to converge and finally spread onto the optimum Pareto front. $N_{g e n}$ depends among other factors on the number of variables to be tuned because the larger the number of variables is a larger $N_{g e n}$ is required with the expense of having longer computational time. In this problem because the number of variables vary according to the number of sensors, $N_{\text {gen }}$ is set at 200 for sensor sets with up to 3 
sensors and for the rest including the full sensor set is set at 250 generations.

In order to achieve the desired limitat ions as described in Section II-B.3 a constraint handling technique is necessary from the ones exists in the literature [3]. The dynamically updated penalty function approach is applied in this paper which has been used by the authors in previous publications. A rigorous description of this constraint handling method can be found in [14]). This method is using penalty functions in order to 'guide' the objective functions in (9) towards the Pareto-Optimality subject to the control limitations shown on Table II. The overall constraint violation function that sums all constraint violations is given as

$$
\Omega\left(k^{(j)}, f^{(i)}\right)=\sum_{j=1}^{J} \omega_{j}\left(k^{(j)}\right)+\sum_{i=1}^{I} \psi_{i}\left(f^{(i)}\right)
$$

where, $\omega_{j}$ is the $j^{\text {th }}$ soft constraint violation for the corresponding $j^{\text {th }}$ quantity to be constrained $(k)$ and $J$ is the total number of soft constraints. Similarly, $\psi$ is the hard constraint violation for the $i^{\text {th }}$ quantity to be constrained $(f)$.

\section{SIMULATION RESULTS AND ANALYSIS}

The overall algorithm is tested in MATLAB R2009b simulation environment without Java function due to large computational need (simulation based). The computer used is the powerful DELL Studio XPS 8100 with Intel(B)Core i7 CPU 870 at $2.93 \mathrm{GHz}$ and 4GB RAM. The average simulation time per sensor set was about 2.7 hours and the procedure for all feasible sensor sets takes around 46 hours. The controller selection criteria $\left(f_{c_{i}}, f_{k}\right)$ for the desired closed-loop response are given as follows

$$
\begin{aligned}
& f_{c_{1}} \equiv \ddot{z}_{r m s} \leq 0.5 \mathrm{~m} / \mathrm{s}^{2}, f_{c_{2}} \equiv u_{n_{r m s}} \leq 10 \mathrm{~V}, \\
& f_{k} \equiv \max (\epsilon)
\end{aligned}
$$

From the simulation results it was found that the proposed systematic framework is able to identify, stable, stabilizing controllers for 11/16 sensor sets while five of them do not satisfy $\Omega$ in (13). However, they could be used if the constraint violation is not critical for the (perhaps degraded) performance of the suspension.

Table III lists some sensor sets selected for a deeper analysis of the simulation results. The second column is the sensor sets and the first the corresponding identification number. The next four columns are the variables from the stochastic closed-loop response while the further four shows the variable values from the deterministic response. The next column is the resulting robustness margin from the $\mathcal{H}_{\infty}$ robust control design and the $12^{\text {th }}$ column lists the resulting RMS level of the noise on the input voltage. The $13^{\text {th }}$ column shows whether the overall constraint violation function, $\Omega$ is satisfied or not. The last column is the SFAR as defined in Section III.

As it can be seen from the $\Omega$, most of the sensor sets listed in Table III satisfy the closed-loop responses constraints. Sensor sets id:4 and 7 violate the stability margin which is why the SFAR is not evaluated (i.e. they will not be considered for control of the EMS) while the rest of the sensor sets including the single air gap measurement shows to satisfy the control requirements of the EMS. The LSDP requires that the air gap is a standard measurement and therefore only one single sensor set exists (id:1) while it is assumed that the particular sensor never fails or simply is has a traditional triple redundancy for fault tolerance. It's SFAR is zero meaning that there is no way to maintain performance if fails. Moving to the id:2-3 there is only one fault scenario where one sensor can fail in each sensor set leaving the air gap measurement the only one to stabilize the suspension giving $100 \%$ sensor fault recovery. Sensor sets id:5,6 (triple sensors) shows to have an SFAR of $66 \%$ which similar to the full sensor set. id:8 and 10 have larger SFAR than id:5,6,9 and 11 (the full sensor set) but less than id:2 and 3. Which means that is not possible to accommodate all possible sensor fault combinations.

Thus, it can be said that either id:2 or 3 are the best sensor sets candidates to control the EMS system with the minimum number of sensors with which optimum performance is achieved while offer sensor fault tolerance.

Simulation results verify the choice. The fault scenario is that while the EMS moves along a gradient of the rail as given in Section II-B the flux density sensor has failed at one second giving a random low frequency characteristics signal as depicted in Fig.4. Figure 5 shows the error of the air gap signals between the nominal and fault situation $\left(z_{t}-z\right)_{e}$. As it can be seen both stability and performance are well maintained after the flux density sensor has failed while the same results appear for the stochastic behaviour of the track.

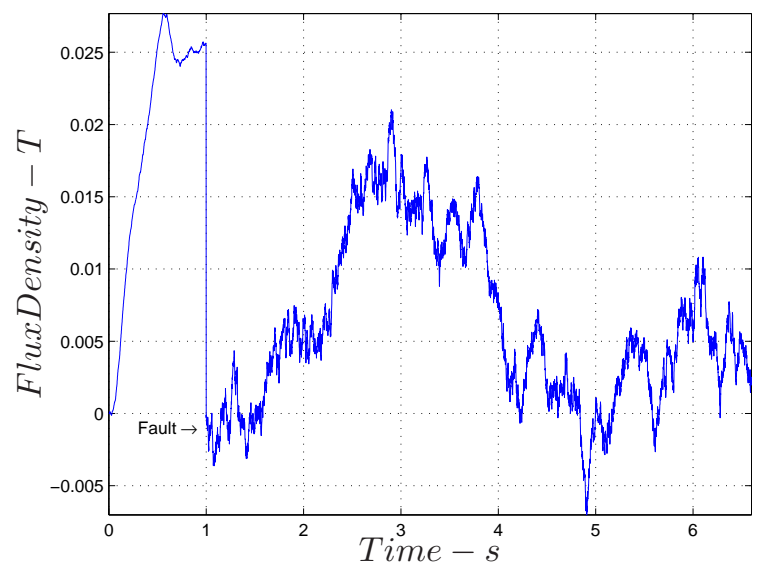

Fig. 4. Faulty flux for the deterministic response.

\section{CONCLUSIONS AND FUTURE WORKS}

It is clear that the presented systematic framework can serve as an efficient tool in order to simplify the optimum sensor selection for fault tolerant control in a model-based control system design. The EMS system has served as a convincing example to test the efficacy of the proposed framework and it was found that two sensors are sufficient to ensure optimal and robust performance as well as offer $100 \%$ 
TABLE III

OPTIMISED SENSOR CONFIGURATIONS USING $\mathcal{H}_{\infty}$ LOOP-SHAPING DESIGN PROCEDURE.

\begin{tabular}{c|c||c|c|c|c||c|c|c|c||c|c|c|c}
\hline \hline & \multicolumn{9}{|c||}{} & \multicolumn{9}{c|}{ Stochastic Input Response } & \multicolumn{2}{|c|}{ Deterministic Input Response } & & & & \\
\hline & & $g_{r m s}$ & $u_{c_{r m s}}$ & $\ddot{z}_{r m s}$ & $i_{r m s}$ & $g_{p}$ & $u_{c_{p}}$ & $t_{s}$ & $e_{s s}$ & $\epsilon$ & $u_{n_{r m s}}$ & $\Omega$ & $S F A R$ \\
\hline & Sensor set & $\mathrm{mm}$ & $\mathrm{V}$ & $m s^{-2}$ & $A$ & $\mathrm{~mm}$ & $\mathrm{~V}$ & $s$ & & & $\mathrm{~V}$ & & $\%$ \\
\hline 1 & $\left(z_{t}-z\right)$ & 1.57 & 47.67 & 0.89 & 1.45 & 1.24 & 11.92 & 2.29 & $\checkmark$ & 0.15 & 0.25 & $\checkmark$ & 0 \\
2 & $b,\left(z_{t}-z\right)$ & 1.56 & 24.54 & 0.47 & 1.24 & 4.64 & 33.94 & 2.10 & $\checkmark$ & 0.20 & 0.39 & $\checkmark$ & 100 \\
3 & $\left(z_{t}-z\right), \dot{z}$ & 1.57 & 47.67 & 0.89 & 1.45 & 1.24 & 11.92 & 2.29 & $\checkmark$ & 0.15 & 0.25 & $\checkmark$ & 100 \\
4 & $\left(z_{t}-z\right), \ddot{z}$ & 1.73 & 34.14 & 0.70 & 1.47 & 1.98 & 16.79 & 2.23 & $\mathrm{x}$ & $\mathbf{0 . 1 1}$ & 0.28 & $\mathrm{x}$ & - \\
5 & $i, b,\left(z_{t}-z\right)$ & 1.38 & 29.18 & 0.47 & 1.11 & 4.36 & 32.36 & 2.15 & $\checkmark$ & 0.22 & 1.01 & $\checkmark$ & 66 \\
6 & $\left.i, z_{t}-z\right), \dot{z}$ & 1.57 & 47.67 & 0.89 & 1.45 & 1.24 & 11.92 & 2.29 & $\checkmark$ & 0.15 & 0.25 & $\checkmark$ & 66 \\
7 & $b,\left(z_{t}-z\right), \dot{z}$ & 3.05 & 39.89 & $\mathbf{1 . 0 5}$ & 2.53 & 1.16 & 10.41 & 2.56 & $\mathrm{x}$ & $\mathbf{0 . 0 5}$ & 0.07 & $\mathrm{x}$ & - \\
8 & $i, b,\left(z_{t}-z\right), \dot{z}$ & 1.58 & 24.16 & 0.47 & 1.25 & 4.74 & 34.67 & 2.10 & $\checkmark$ & 0.20 & 0.35 & $\checkmark$ & 71 \\
9 & $i, b,\left(z_{t}-z\right), \ddot{z}$ & 1.38 & 29.19 & 0.47 & 1.11 & 4.36 & 32.35 & 2.15 & $\checkmark$ & 0.22 & 1.01 & $\checkmark$ & 57 \\
10 & $i,\left(z_{t}-z\right), \dot{z}, \ddot{z}$ & 1.37 & 29.59 & 0.49 & 1.12 & 4.24 & 31.51 & 2.14 & $\checkmark$ & 0.21 & 0.99 & $\checkmark$ & 71 \\
11 & $i, b,\left(z_{t}-z\right), \dot{z}, \ddot{z}$ & 1.33 & 31.68 & 0.49 & 1.10 & 4.14 & 30.87 & 2.15 & $\checkmark$ & 0.23 & 1.07 & $\checkmark$ & 62 \\
\hline \hline
\end{tabular}

$g_{p} \equiv\left(z_{t}-z\right)_{p}, g_{r m s} \equiv\left(z_{t}-z\right)_{r m s}, e_{s s} \equiv\left(z_{t}-z\right)_{e_{s s}}$

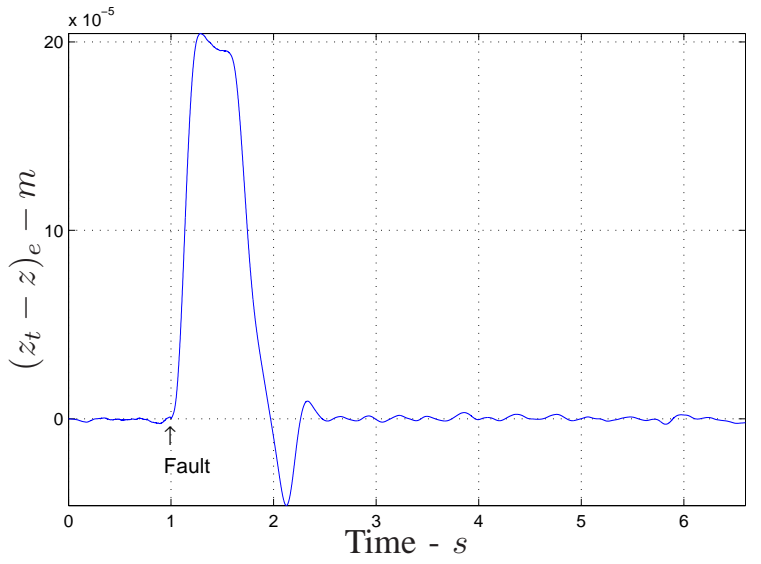

Fig. 5. Error between airgap with healthy and faulty flux at $1 s$.

fault recovery.Although time consuming the framework is very flexible and can be used for more complex systems than the EMS system.

\section{REFERENCES}

[1] M. Blanke, R. Izadi-Zamanabadi, S. A. Bogh, and C. P. Lunau. Fault-tolerant control systems - a holistic view. Control Engineering Practice, 5(5):693-702, 1997.

[2] M. Blanke, M. Kinnaert, J. Lunze, and M. Staroswiecki. Diagnosis and Fault-Tolerant Control. Springer-Verlag New York, Inc., Secaucus, NJ, USA, 2003.

[3] C. A. C. Coello. Theoretical and numerical constraint-handling techniques used with evolutionary algorithms: A survey of the state of the art. Computer Methods in Applied Mechanics and Engineering, 191(11-12):1245-1287, 2002.

[4] Kalyanmoy Deb, Amrit Pratap, Sameer Agarwal, and T. Meyarivan. A fast and elitist multiobjective genetic algorithm: Nsga-ii. IEEE Transactions on Evolutionary Computation, 6(2):182-197, 2002.

[5] P. J. Fleming and R. C. Purshouse. Evolutionary algorithms in control systems engineering: A survey. Control Engineering Practice, 10(11):1223-1241, 2002.

[6] R. M. Goodall. Dynamic characteristics in the design of maglev suspensions. Proceedings of the Institution of Mechanical Engineers, Part F: Journal of Rail and Rapid Transit, 208(1):33-41, 1994.

[7] R. M. Goodall. Dynamics and control requirements for ems maglev suspensions. In Proceedings on international conference on Maglev, pages 926-934, 2004.

[8] R. M. Goodall. Generalised design models for ems maglev. In Proceedings of MAGLEV 2008 - The $20^{\text {th }}$ International Conference on Magnetically Levitated Systems and Linear Drives, 2008.
[9] R. M. Goodall. The theory of electromagnetic levitation. Physics in Technology, 16(5):207-213, Sep 1985.

[10] R. Isermann. Supervision, fault-detection and fault-diagnosis methods-an introduction. Control Engineering Practice, 5(5):639652, 1997.

[11] Abdullah Konak, David W. Coit, and Alice E. Smith. Multi-objective optimization using genetic algorithms: A tutorial. Reliability Engineering and System Safety, 91(9):992-1007, 2006.

[12] Hyung-Woo Lee, Ki-Chan Kim, and Ju Lee. Review of maglev train technologies. IEEE Transactions on Magnetics, 42(7):1917-1925, 2006.

[13] D. C. McFarlane and K. Glover. A loop-shaping design procedure using $h_{\infty}$ synthesis. IEEE Transactions on Automatic Control, 37(6):759-769, 1992.

[14] K. Michail. Optimised configuration of sensing elements for control and fault tolerance applied to an electro-magnetic suspension system, 2009. PhD dissertation, Loughborough University, Department of Electronic and Electrical Engineering. http://hdl.handle.net/2134/5806.

[15] K. Michail, Y. Zhou, A. Zolotas, R. Goodall, and G. Halikias, Optimised sensor configurations with reduced order controllers applied to an ems system. In $29^{\text {th }}$ Chinese Control Conference, pages 3595$3600,2010$.

[16] K. Michail, A. Zolotas, and R. Goodall. Simulation-based optimum sensor selection design for an uncertain ems system via monte-carlo technique. International Federation of Automatic Control $18^{\text {th }}$ World Congress, 2011. [Under Review].

[17] K. Michail, A. Zolotas, and R. M. Goodall. Optimised sensor configurations for a maglev suspension. Proceedings of the $17^{\text {th }}$ World Congress The internetional Federation of Automatic Control, pages 8305-8310, July 6-11, 2008.

[18] K. Michail, A. C. Zolotas, R. Goodall, and J. T. Pearson. Sensor optimisation via $h_{\infty}$ applied to a maglev suspension system. International Journal of Computer Systems Science and Engineering, 5(1):43-49, 2009.

[19] K. Michail, A. C. Zolotas, and R. M. Goodall. Ems systems: Optimised sensor configurations for control and sensor fault tolerance. Japan Society of Mechanical Engineers, International Symposium on Speedup, Safety and Service Technology for Railway and Maglev Systems, 2009.

[20] K. Michail, A. C. Zolotas, R. M. Goodall, and J. T. Pearson. Maglev suspensions - a sensor optimisation framework. In $16^{\text {th }}$ Mediterranean Conference on Control and Automation, pages 1514-1519, June 25-27 2008.

[21] R. J. Patton. Fault-tolerant control: The 1997 situation. In IFAC Symposium on Fault Detection Supervision and Safety for Technical Processes, volume 3, pages 1029-1052, 1997.

[22] S. Skogestad and I. Postlethwaite. Multivariable Feedback Control Analysis and Design. John Wiley \& Sons Ltd, $2^{\text {nd }}$ Edition, New York, 2005.

[23] Youmin Zhang and Jin Jiang. Bibliographical review on reconfigurable fault-tolerant control systems. Annual Reviews in Control, 32(2):229252, 122008. 